\title{
A possible evolutionary role of formaldehyde
}

\author{
Miklós Péter Kalapos \\ Theoretical Biology Research Group, Budapest, Hungary \\ Fax, $+36-1-3975225$
}

Accepted 2 March 1999

\begin{abstract}
Formaldehyde is a compound which is believed to have had a role in evolutionary processes. On the other hand, the (methyl)glyoxalase pathway is a route being present in all biological organisms whereas its function has not yet been recognized in the biochemical machinery. In this article it is raised that (methyl)glyoxalase path might have functioned as a bridge between formose and archaic reductive citric acid cycles in surface metabolists at the early stage of evolution. According to the theory, formaldehyde was essential for the mentioned system as a raw molecule. Based on thermodynamic calculations a simple way of regulation is also shown. The simplicity of the theory may be in a good agreement with and an explanation of why the (methyl)glyoxalase system is of ubiquitous nature.
\end{abstract}

Keywords: formaldehyde, methylglyoxal, glyoxalase, surface metabolism, reductive citric acid cycle

\section{Introduction}

The enzymes have a well defined metabolic function in the biochemical machinery of those living organisms in which they appear. This rule has to apply to such a widespread enzyme system like glyoxalases, too. Eventhough since the discovery of the glyoxalase system its role has been investigated very thoroughly the significance of glyoxalases in the biological network is still unclear (Kalapos, 1994). This is true even if almost everything is known about the biochemistry and molecular biology of glyoxalases (Vander Jagt, 1989). The ubiquitous nature and the presence of the glyoxalase system in all living organisms should predictate an important but as yet unknown role for this route in the biological network, perhaps in the early stages of evolutionary process.

The aim of this paper is to propose an answer to this almost hundred year old puzzle of biochemistry. Namely, what the biological role of methylglyoxalase pathway may have been in the course of evolution.
However, before turning to the description of the possible evolutionary role of methylglyoxalase pathway it is appropriate to stress three sets of facts which finally led to the suggession of the theory.

\section{Antecendents and problems}

\section{The glyoxalase system}

There is an ubiquitous enzyme system, the so-called glyoxalase system, which is found in almost all the species and tissues investigated until now (Vander Jagt, 1989). The glyoxalase system consists of two enzymes, glyoxalase I (E.C. 4.44.1.5., lactoylglutathione lyase) and glyoxalase II (E.C. 3.1.2.6., hydroxyacyl glutathione hydrolase), and consumes a catalytic amount of reduced glutathione (Mannervik, 1980; Vander Jagt, 1989). The catalytic function of this enzyme system is to convert $\alpha$ oxoaldehydes into their corresponding $\alpha$ hydroxicarboxylic acid counterparts in two sequential steps via the inter-mediates S-D-acyl-glutathione derivatives (Racker, 1951). Nevertheless, the problem is that the biological function of this enzyme system in biochemical machinery is still obscure.

The glyoxalase enigma has adhered the interest of several out of the brilliant biochemists of our century, without giving a complete list of the scientists here the names of Hopkins, Neuberg, Lohmann, Racker and Szent-Györgyi are mentioned. The most influential theories in this regard are listed in the Table I. However, despite the endeavours and suggestions the problem has remained open since either of the theories proved to be able to give the exact answer to this long lasting problem emerged concerning the function of this pathway. Because as depicted in the Embden-Meyerhof scheme of glucose breakdown, the main stream of glycolysis operates in another way, through the three carbonphos-phates, thus glyoxalase pathway is believed to have a subordinate role in glycolysis (Meyerhof, 1948). Further-more, the clarification of the role of glyoxalases in cell division regulation, as envisaged by Szent-Györgyi, needs further experimental supports while the theory itself suffers from intramural contradictions, as well (Kalapos, 1998). And the role of the glyoxalase pathway in detoxi-cation eventhough exactly describes a function for the glyoxalases (Aronsson and Mannervik, 1977), it does not give the reason of why it ought to be of ubiquitous nature (Kalapos, 1994). So the first starting point to our suggestion is that there is a widespread enzyme system without any recognized function in the cells. 


\section{Surface metabolism}

The second branch of the problems is related to the prebiological evolution. As far as it is known, Oparin was the first in the 1920s, who presented a comprehensive concept on the emergence of cellular life. The primeval soup theory of Oparin was though at that time as to be an answer to how the organization of matter into living might have started. Without going into details in this regard since the literature on the theory of Oparin is unaccountable, it has to be stressed that one of the most crucial problems of the primeval soup theory is that the primeval ocean as a solution of organic compounds should have been very diluted retaining the probability of bumping of different molecules very low. To overcome this obstacle, Wachtershauser presented a new hypothesis for prebiological evolution by refusing the primeval soup theory and by introducing the concept of the biochemistry of surface bound molecules (Wachtershauser 1988, 1990). The most important statements of surface meta-bolism theory are as follows: (i) the prebiological soup theory (Oparin's theory) has to be refused since the primeval soup, if existed, should have been a very diluted solution of organic compounds; (ii) the organic compounds had to be enchored to a charged surface; (iii) the meta-bolic network was a chemoautotrophic system in which the source of energy for carbon fixation was provided by pyrite formation; (iv) the reductive citric acid cycle was a nonenzymic archaic precursor cycle; ( $v$ ) the constituents of the cycle coexisted in an equilibrium with their thioanalogues; and finally (vi) the anaplerotic route for the cycle operated through one of the following pathways: acetyl-CoA pathway, glycine synthetase pathway or the oxalate route (Wachtershauser 1990; Maden, 1995). Indeed, from biochemical point of view it is obvious that small, simple molecules had been needed as prerequisites to any function for the ignition of the cycle, that is the anaplerotic route for the cycle is very critical. In this regard the surface metabolism theory has been insufficiently worked out (Kalapos, 1997).

As proposed in the surface metabolism theory (Wachtershauser 1988, 1990), the first source of energy for life would have been the pyrite formation in the reaction depicted in the Equation (1):

$$
\begin{array}{r}
\mathrm{FeS}+\mathrm{H}_{2} \mathrm{~S} \rightarrow \mathrm{FeS}_{2} \text { (pyrite) }+\mathrm{H}_{2} \quad(-38 \mathrm{~kJ} / \mathrm{mol}) \\
\left(\mathrm{pH} 7, \mathrm{~T}=25^{\circ} \mathrm{C}, 1 \mathrm{M} \mathrm{H}_{2} \mathrm{~S}\right)
\end{array}
$$

It is also to be noticed that beside the above mentioned problem concerning the anaplerosis there are several other critical points too, e.g. thermodynamic and kinetic aspects of surface metabolism or the coupling system providing link between sulfide oxidation and the assembly of reactions (de Duve and Miller, 1991; Wactershauser, 1994). However, the discussion of the above mentioned problems is out of the scope of the paper.

\section{Formaldehyde}

The third starting point is the well known formose cycle. And with this cycle we have arrived at formaldehyde. Formaldehyde is one of the most abundant organic compounds in the intrastellar space and commets (Navarro-Gonzalez, 1992). It can also be produced in laboratories under so-called prebiotic conditions (Miller, 1987). And it is a simple and reactive compound. Furthermore, through formose reaction it leads to the generation of a wide variety of carbohydrates, therefore, it is an ideal chemical to take place in the early stage of evolution.

\section{The theory}

The theory is that the early methylglyoxalase pathway functioning without any participation of enzymes was the link between the formose cycle and the reductive citric acid cycle of surface metabolists thus providing a link between these cycles and a fuel for the citric acid cycle. Probably this fact might be an explanation of why this pathway is of ubiquitous.

\section{Flow of matter from formaldehyde to archaic citric acid cycle}

As shown in the Figure 1 (reaction a), the interaction of two molecules of formaldehyde leads to the formation of glycolaldehyde (Breslow, 1959). It is believed that glycolaldehyde is autocatalytic to its own formation and in the formose cycle an additional molecule of glycolaldehyde is produced from two other molecules of formaldehyde (Breslow, 1959). Both dihydroxyacetone and glyceralde-

Table 1. The history of roles suggested for glyoxalase system.

\begin{tabular}{lcc}
\hline Suggested role for the glyoxalases & The year when the role was suggested & Reference \\
\hline Participation in the glucose breakdown & 1926 & Toennissen und Fischer, 1926 \\
Regulation of cell division & 1965 & Szent-Györgyi, 1965 \\
Detoxication of $\alpha$-oxoaldehydes & 1977 & Aronsson and Mannervik, 1977 \\
Participation in microtubule assembly & 1978 & Gillespie, 1978 \\
\hline
\end{tabular}




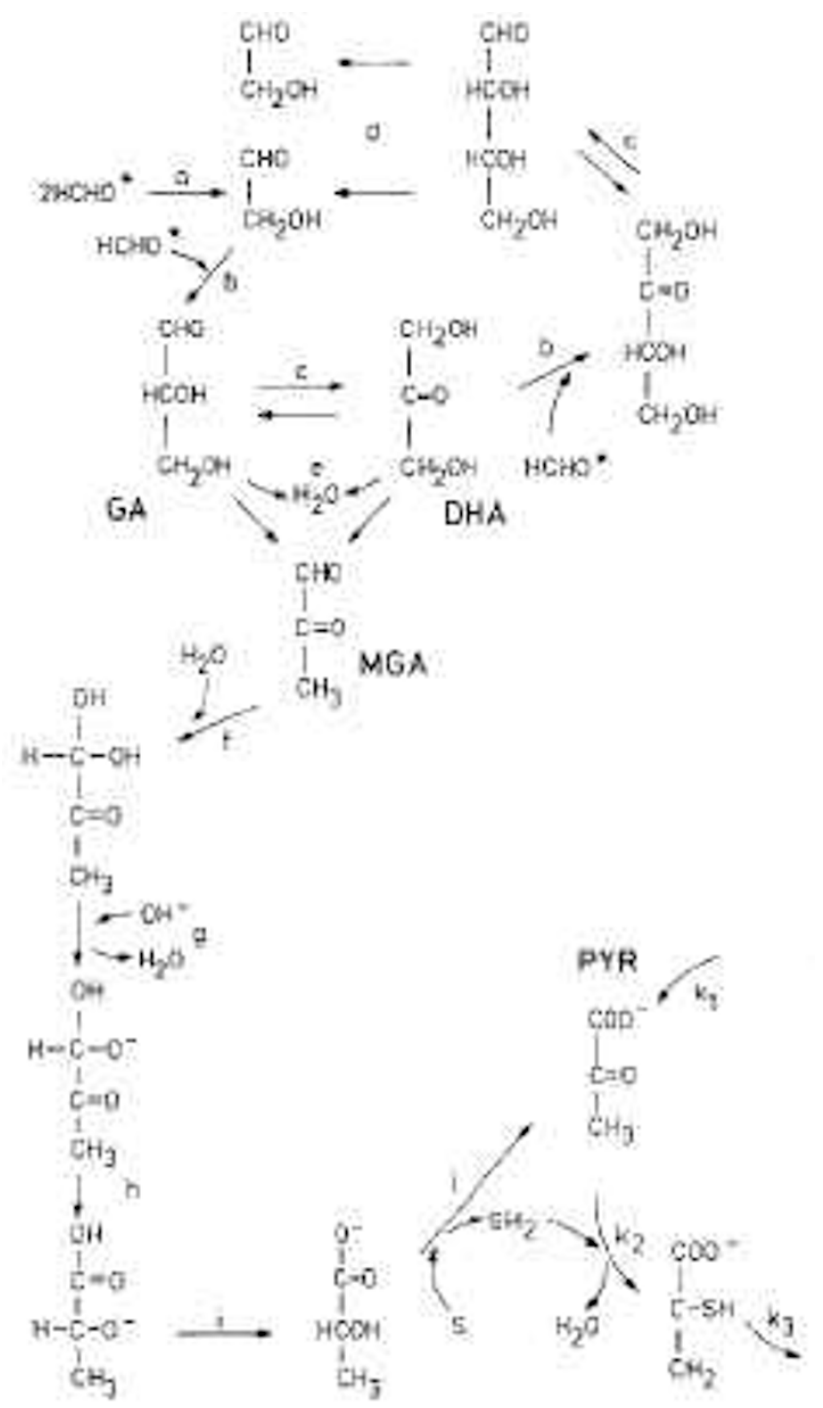

Figure 1. Reactions that lead to pyruvate formation from formaldehyde. a, selfcondensation; $b$, aldol-condensation; $c$, carbonyl shift isomerization; $d$, aldolretroaldol reaction; e, dehydration; $f$, hydration; $g$-i, suggested disproportionation of methylglyoxal to lactate in which reaction; $h$ is slow while reaction. $\mathrm{i}$ is fast (Fedoronko and K'nigstein, 1971); j, oxidation linked to $\mathrm{H}_{2} \mathrm{~S}$ formation, $\mathrm{k}_{1-3}$, reactions in the archaic reductive citric acid cycle as defined in the literature (Wachtershauser, 1990). Asterisks indicate the molecules of formaldehyde that should be prebiotically synthesized in the surroundings; PYR, pyruvate; MGA, methylglyoxal; GA, glyceraldehyde; DHA, dihydroxyacetone. Taken from (Kalapos, 1997) with the permission of the publisher. hyde are intermediates of the formose cycle. The formation of methylglyoxal from either glyceraldehyde or from dihydroxiacetone is possible (Figure 1. reaction e) (Fedoronko and Königstein, 1969). The reaction itself is an acid-base catalyzed dehydration (Fedoronko and Königstein, 1969). Thus, as an offshoot of the formose cycle methylglyoxal can be generated.

The conversion of methylglyoxal into lactate is an intramolecular Cannizzaro reaction (Figure 1). Methylglyoxal is first hydrated and then attacked by a hydroxy group resulting in a giving down of one molecule of water (Figure 1, reactions $f$ and $g$ ). The subsequent steps are not fully corroborated, but it seems that lactate is resulted from the reaction (Figure 1, reactions $h$ and $i$ ) (Fedoronko and Königstein, 1969, 1971).

A critical point is the conversion of lactate into pyruvate
(Figure 1, reaction $j$ ). In the extant metabolism, the lactate/pyruvate and $\mathrm{NADH}+\mathrm{H}^{+} / \mathrm{NAD}^{+}$redox-systems have the standard reduction potentials of $\varepsilon_{0}{ }^{\prime}=-0.19 \mathrm{v}$ and $\varepsilon_{0}{ }^{\prime}=-0.32 v$, respectively (Elödi, 1989). From the values of standard reduction potentials it follows that on its own the equilibrium of the reaction between pyruvate and lactate is shifted towards L-lactate formation.

Under standard conditions the $\mathrm{FeS} / \mathrm{H}_{2} \mathrm{~S}$ system has a standard reduction potential of $\varepsilon_{0}^{\prime}=-0.62 \mathrm{v}$ that corresponds to a standard free energy of $\Delta \mathrm{G}_{0}{ }^{\prime}=-38 \mathrm{~kJ} / \mathrm{mol}$ (Maden, 1995). By this system the oxidation of lactate to pyruvate is not possible, since the standard reduction potential of lactate/pyruvate system is the equivalent of a standard reduction potential of $\varepsilon_{0}^{\prime}=-0.19 \mathrm{v}$ (Elödi, 1989), namely, the equilibrium would considerably be shifted towards lactate. None the less, considering the facts 
that on the one hand, the $\mathrm{S}^{\circ} / \mathrm{H}_{2} \mathrm{~S}$ system has a standard reduction potential of $\varepsilon_{0}{ }^{\prime}=-0.24 \mathrm{v}$ (Thauer, 1988) and on the other hand, the reduction of elementary sulfur to $\mathrm{H}_{2} \mathrm{~S}$ can be connected to the oxidation of lactate to pyruvate as shown in the Equation (2):

lactate $+\mathrm{S}^{0} \Rightarrow$ pyruvate $+\mathrm{H}_{2} \mathrm{~S} \quad(2)$,

then there is a possibility for the reaction. In the reaction the standard free energy change calculated on the basis of reduction potentials presented, comes about $\Delta \mathrm{G}_{0}{ }^{\prime}=$ $+9.62 \mathrm{~kJ} / \mathrm{mol}$, which corresponds to an equilibrium constant of 0.02 for the reaction and it is smaller than the free energy change $(+25.2 \mathrm{~kJ} / \mathrm{mol})$ determined for the interconversion of L-lactate into pyruvate when nicotinamide dinucleotide is present as hydrogen acceptor (Elödi, 1989). From all of these it follows that a near equilibrium situation can be reached which, similarly to the situation seen in the extant metabolism, can be enough to make the reaction proceed towards pyruvate formation if both pyruvate and $\mathrm{H}_{2} \mathrm{~S}$ are consumed. Since $\mathrm{H}_{2} \mathrm{~S}$ takes a part in pyrite formation and pyruvate enters the archaic reductive citric acid cycle, this requirement is easy to be met.

\section{Concluding remarks}

To sum up, the ubiquitous nature of methylglyoxalase pathway suggests an important role for this pathway in the evolution. This role for the methylglyoxalase pathway would have been to operate as an anaplerotic route for the reductive citric acid cycle of surface metabolists at the early stage of evolution. In the theory formaldehyde has a central role in metabolic network serving as a raw molecule for the formose cycle.

It is a general feature of evolution that it preserves molecular mechanisms and uses them for different purposes when the innovation proved successful. Probably this has happened to glyoxalases, as well. Having lost its essential evolutionary role in the late metabolism the current function of glyoxalase path is feasibly restricted only to the detoxication of $\alpha$-oxoaldehydes, especially to the detoxication of methylglyoxal inevitably produced during glycolysis and aminoacetone cycle. Indeed, this is the fact that makes glyoxalases possible targets for the investigations dealing with the development of diabetic complications.

\section{Acknowledgements}

This research was made possible by the grant from the National Scientific Research Found (OTKA), Budapest, Hungary ( $T$ 022914). The paper was presented in part before the audience of the Fourth International Conference on The Role of Formaldehyde in Biological Systems held July 1-4, 1998. in Budapest, Hungary.

\section{References}

Aronsson, A. C. and Mannervik, B. (1977) Characterization of glyoxalase I purified from pig erythrocytes by affinity chromatography. Biochem. J. 165: 503-509

Breslow, R. (1959) On the mechanism of the formose reaction. Tetrahedron Lett. 21: 22-26 de Duve, C. and Miller, S.L. (1991) Two-dimensional life? Proc. Natl. Acad. Sci. USA 88: 10014-10017

\section{Elödi, P. (1989) Biokémia,Akadémia Kiadó, Budapest}

Fedoronko, M. and Königstein, J. (1969) Kinetics of mutual isomerization of trioses and their dehydration to methylglyoxal. Collection Czechoslov. Chem. Commun. 34: 38813894

Fedoronko, M. und Königstein, J. (1971) Kinetik und Mechanismus der Disproportionierung von Methylglyoxal zu Milchsaure. Collection Czecho-slov. Chem. Commun. 36: 3424-3430

Gillespie, E. (1978) Concanavalin A increases glyoxalase enzyme activities in polymorphonuclear leukocytes and lymphocytes. J. Immun. 121: 923-925

Kalapos, M. P. (1994) Methylglyoxal toxicity in mammals. Tox. Letters 73: 3-24

Kalapos, M. P. (1997) Possible evolutionary role of methylglyoxalase pathway/Analplerotic route for reductive citric acid cycle of surface metabolists, $\mathrm{J}$. Theor. Biol. 188: 201-206

Kalapos, M. P. (1999) On the promine/retine theory of cell division. Biochim. Biophys. Acta 1426: 1-16

Maden, B. E. H. (1995) No soup for starters? Autotrophy and origins of metabolism. Trends Biochem. Sci. 20: 337-341

Mannervik, B. (1980) Glyoxalase I. In Enzymatic basis of detoxication. Vol. II. (Jakoby, W.B. ed.), pp. 263-273, Academic Press

Meyerhof, O. (1948) New investigations of enzymatic glycolysis and phosphorylation. Experientia 4: 169-176

Miller, S.L. (1987) Which organic compounds could have occured on the prebiotic Earth? Cold Spring Harb. Symp. Quant. Biol. 52: 17- 26

Navarro-Gonzalez, R. (1992) Role of formaldehyde in the origin of life. In Proceedings of the 3rd International Conference on Role of Formalde-hyde in Biological systems. (Tyihák, E. ed.) pp. 93-100, Hungarian Bio-chemical Society

Racker, E. (1951) The mechanism of action of glyoxalase. J. Biol. Chem. 190: 685-696 Szent-Györgyi, A. (1965) Cell division and cancer. Science (Washington D.C.) 149: 34-

Thauer, R. K. (1988) Citric-acid cycle, 50 years on. Eur. J. Biochem. 176: 497-508

Toenniessen, E. und Fischer, W. (1926) Methylglyoxal als Abbauprodukt der Glykolyse. Zeitschr.physiol.Chem. 161: 254-264

Vander Jagt, D. L. (1989) The glyoxalase system. In Glutathione: Chemical, Biochemical and Medical Aspects - part A. (Dolphin, D., Poulson, R. and Avramovic, O. eds.) pp. 597-641, John Wiley and Sons, New York

Wachtershauser, G. (1988) Before enzymes and templates: Theory of surface metabolism. Microbiological Rev. 52: 452-484

Wachtershauser, G. (1990) Evolution of the first metabolic cycles. Proc. Natl. Acad. Sci. USA 87: 200-204

Wachtershauser, G. (1994) Life in a ligand sphere. Proc. Natl. Acad. Sci. USA 91: 42834287 